\title{
Immunohistochemical Evaluation and Prognostic Value of Monocarboxylate Transporter 1 (MCT1) and 4 (MCT4) in T-cell Non-Hodgkin Lymphoma
}

\section{Hu Zhao}

Central South University Xiangya School of Medicine

\section{Yuan Chen}

Central South University Xiangya School of Medicine

\section{You-Ping Liao}

Central South University Xiangya School of Medicine

\section{Hai-Mei Chen}

Central South University Xiangya School of Medicine

\section{Qiu-Hong Yang}

Central South University Xiangya School of Medicine

\section{Yin Xiao}

Central South University Xiangya School of Medicine

Jing Luo

Central South University Xiangya School of Medicine

\section{Zhen-Zhen Chen}

Central South University Xiangya School of Medicine

\section{Lai Yi}

Central South University Xiangya School of Medicine Guo-Yu Hu ( $\sim$ doctor.hgy@163.com )

Central South University Xiangya School of Medicine https://orcid.org/0000-0001-6916-9814

\section{Research Article}

Keywords: T-cell non-Hodgkin lymphoma, monocarboxylate transporter, immunohistochemistry, lactate metabolism

Posted Date: October 29th, 2021

DOI: https://doi.org/10.21203/rs.3.rs-1002043/v1

License: (c) (1) This work is licensed under a Creative Commons Attribution 4.0 International License. Read Full License 
Version of Record: A version of this preprint was published at Clinical and Experimental Medicine on March 3rd, 2022. See the published version at https://doi.org/10.1007/s10238-022-00805-4. 


\section{Abstract}

Tumor cells often exhibit the Warburg effect, wherein, they preferentially undergo glycolysis over oxidative phosphorylation for energy production. Monocarboxylate transporter 1 (MCT1) and 4 (MCT4) are critical symporters mediating lactate efflux and preventing intracellular acidification during tumor growth. Numerous studies have focused on inhibiting MCT1 or MCT4 in various cancers. However, its role in T-cell lymphoma (TCL) is not yet investigated owing to the low incidence of TCL. This study was designed to investigate the expression of MCT1/MCT4 in patients with TCL and determine their prognostic value in this cancer. We performed immunohistochemistry to evaluate the expression level of MCT1/MCT4 in 38 TCL tissue samples and then compared their expression among different TCL subgroups, which were formed based on different clinical characteristics. Survival analysis was performed to evaluate the relationship between MCT1/MCT4 expression and both overall survival (OS) and progression-free survival (PFS). Our results revealed that MCT1 and MCT4 expression was significantly increased in TCL tissues compared to the control group. In addition, increased MCT1 expression associated with the female sex, advanced disease stage, increased serum LDH, Ki-67 at $\geq 50 \%$, and intermediate or high-risk groups as categorized by the International Prognostic Index (IPI) score. We also found that increased MCT1 expression may be associated with reduced OS and PFS. In conclusion, MCT1 and MCT4 are overexpressed in patients with TCL, and may predict poor prognosis. MCT1 inhibition might be a novel treatment strategy for TCL, and further preclinical trials are required.

\section{Introduction}

T-cell lymphomas (TCLs) comprise a group of highly heterogeneous lymphatic neoplasms derived from mature T- and natural killer (NK) cells with aggressive courses and poor prognosis[1]. TCL accounts for approximately $10 \%$ of all non-Hodgkin lymphomas (NHLs) in developed countries[2], with the figure likely to be higher (about 15-20\%) in the Far East[3, 4]. In 2016, according to World Health Organization's (WHO) classification of lymphoid neoplasms, TCL was split into 27 different pathological types based on morphology, immunohistochemistry (IHC), and genetic characteristics[5]. The most common types include peripheral T-cell lymphoma, not otherwise specified (PTCL, NOS), angioimmunoblastic T-cell lymphoma (AITL), ALK-positive anaplastic large-cell lymphoma ( $\left.A L K^{+} A L C L\right)$, ALK-negative anaplastic large-cell lymphoma (ALK ${ }^{-}$ALCL), extranodal NK/T-cell lymphoma, nasal type (ENKTL), cutaneous T-cell lymphoma (CTCL), and mycosis fungoides (MF). The 5-year overall survival (OS) rate for the majority of patients with TCLs treated with traditional first-line therapies, such as cyclophosphamide, doxorubicin, vincristine, and prednisolone (CHOP) and CHOP-like regimens, is only 38.5\%[6-8], and stem cell transplantation (SCT) is not recommended in elderly patients or those with poor physical tolerance[9]. Although researchers have spared no effort to improve the prognosis of TCL, the outcome remains disappointing. Consequently, it is imperative to invent and identify new treatment approaches for TCL.

Tumor cells prefer production of energy via glycolysis and not oxidative phosphorylation; this is referred to as the Warburg effect, which results in increased glucose consumption and an increase in intracellular lactate[10,11]. To maintain lactate homeostasis and prevent intracellular acidification, tumor cells efflux 
the excess lactate via the monocarboxylate transporters (MCTs). MCTs, encoded by the solute carrier 16 (SLC16) family of genes, which share characteristic sequence motifs, act as both cell and mitochondrial membrane-localized transporters and can bidirectionally transfer monocarboxylates (mainly lactate and pyruvate) via passive transport based on the local concentration gradient[12]. Only four of its isoforms (MCT1, MCT2, MCT3, and MCT4) are proton-linked, which is critical for conveying the substrates. MCT1 (SLC16A1) is widely expressed in various tissues and promotes the import and export of several lactates. MCT4 (SLC16A3) has the lowest affinity for lactate and exports lactate from glycolytic cells, including astrocytes, immune cells, chondrocytes, white skeletal muscle fibers, hypoxic cells, and tumor cells[13, 14]. Many studies have reported the expression of both MCT1 and MCT4 in cancer cell lines and different solid tumors, and some of these studies report that MCT1 or MCT4 is upregulated during tumor progression from normal to neoplastic epithelium[15, 16]. In addition, previous cell and mouse experiments revealed that inhibiting MCT1 with an inhibitor or siRNA disrupts the metabolic symbiosis between the oxygenated and hypoxic tumor cells causing the hypoxic (or glycolytic) tumor cells to die from glucose starvation delaying tumor growth $[17,18]$. This means that MCT inhibitors have been identified as potential targeted therapeutic drugs in the treatment of several cancers. Notably, a phase I clinical trial (NCT01791595) of the MCT1 selective inhibitor, AZD3965, in the treatment of adult solid tumors, diffuse large B-cell lymphoma, and Burkitt lymphoma is underway in the UK[19]. There are several related studies evaluating the use of MCT inhibitors in hematological malignancies, particularly focusing on preclinical evaluations of their utility in B-cell non-Hodgkin lymphomas[20-23]. However, only two previous studies have investigated the expression levels of these MCTs in patient derived tissue samples[24, 25]. Furthermore, studies evaluating the expression and prognostic value of MCT1 and MCT4 in patients with T-cell non-Hodgkin lymphoma are rare.

In this study, we collected tissues samples from 38 cases of TCL, and quantified IHC expression data of MCT1 and MCT4 from the pathological tissues was then compared with the control group. The differences in their expression levels were then correlated with various clinical characteristics to reveal the clinicopathological significance of MCT1 and MCT4 expression in TCL. We also analyzed the relationship between the different expression levels of these two symporters and the prognosis of patients with TCL. Our findings make a significant contribution to the investigation of MCT1 inhibitors as potential targets for therapeutics in TCLs.

\section{Materials And Methods}

\section{Gene expression analysis using data from the Cancer Cell Line Encyclopedia (CCLE)}

The CCLE (https://portals.broadinstitute.org/ccle)[26] is a collaborative project that provides open access to genomic data, analysis, and visualization of about 1000 cell lines. We visualized the gene expression data of MCT1 (SLC16A1) and MCT4 (SLC16A3) in T-cell lymphoma cell lines as box plots using the CCLE platform and compared their expression levels to those of other cancer cell lines. 


\section{Patients and tissue samples}

We enrolled 38 patients diagnosed with TCL in this study. These patients were all receiving treatment at the Affiliated Zhuzhou Hospital, XiangYa School of Medicine, Central South University (Hunan, China) between July 2015 and November 2019. Our cohort included 13 cases of ENKTL, 10 cases of PTCL-NOS, eight cases of AITL, two cases of T-lymphoblastic lymphoma (T-LBL), two cases of ALK ${ }^{+} A L C L$, and one case each of $A L K^{-} A L C L$, follicular T-cell lymphoma (FTL), and monomorphic epitheliotropic intestinal Tcell lymphoma (MEITL). Randomly selected 30 samples from patients with reactive lymphoid hyperplasia (RLH) served as the control group. Patients with TCL were then sub-grouped based on various clinicopathological parameters, namely: gender, age, pathological diagnosis, performance status of Eastern Cooperative Oncology Group (ECOG) score, Ann Arbor stage, serum lactate dehydrogenase (LDH) level, extent of their extranodal involvement, International Prognostic Index (IPI) score, the presence or absence of bone marrow involvement, Ki-67 index, serum beta 2-microglobulin ( $\beta 2-\mathrm{MG}$ ) level and the presence or absence of the "B" symptoms. Formalin-fixed paraffin-embedded (FFPE) pathological tissues were collected from the hospital's pathology department and the sections were stained with hematoxylin and eosin (HE). The use of tissue specimens was approved by the institutional review board, and the study was conducted under the ethical guidelines described in the Declaration of Helsinki. Patient followup for this cohort continued until April 2021 with three independent hematologists who were not affiliated with this study. Overall survival (OS) was defined as the interval between enrollment and death or final observation. Progression-free survival (PFS) was defined as the interval between enrollment and the first signs of tumor progression, death, or the final observation in the follow up time frame. The initial evaluation, staging, and response definition for each patient with TCL were based on the recommendations of the Lugano classification of lymphoma criteria[27].

\section{Immunohistochemistry (IHC)}

Paraffin tissue sections were heated at $65^{\circ} \mathrm{C}$ for $2 \mathrm{~h}$, deparaffinized in xylene for $15 \mathrm{~min}$, cleaned with $100 \%$ ethanol, and then rehydrated using decreasing concentrations of ethanol $(95 \%, 85 \%$, and $75 \%$ for 5 min each). Endogenous peroxidase activity was blocked by incubation with $3 \% \mathrm{H}_{2} \mathrm{O}_{2}$ for 15 min, and epitope retrieval was induced by heating the samples in citrate buffer $(\mathrm{pH} 6.0)$ under high pressure for 3 min before the slides were cooled in cold water for $10 \mathrm{~min}$. Non-specific binding was blocked by incubating each sample with $100 \mu \mathrm{L}$ goat serum at $20-25^{\circ} \mathrm{C}$ for $60 \mathrm{~min}$. The slides were immersed in phosphate-buffered saline (PBS, pH 7.4) prior to each of the above steps to prevent cross-over and reagent contamination. The blocking buffer was then removed, antibodies were added, and the cells were incubated at $4^{\circ} \mathrm{C}$ overnight. The primary antibodies used were as follows: rabbit polyclonal antibody for MCT1 (20139-1-AP; Proteintech, USA; 1:600 dilution, cytoplasmic and membrane staining) and rabbit polyclonal antibody for MCT4 (22787-1-AP; Proteintech, USA; 1:200 dilution, membrane staining). The dilution ratios were validated using a pre-experiment; slides without the primary antibodies served as negative controls in these assays. After washing in PBS supplemented with Tween 20 (PBST), the sections were incubated with horseradish peroxidase (HRP)-labeled secondary antibody (S0001; Affinity Biosciences, USA; 1:200 dilution) for 30 min and then visualized with 3,3'-Diaminobenzidine (DAB). These 
samples were then counterstained with hematoxylin for $15 \mathrm{~min}$, followed by differentiation in $1 \%$ acid alcohol and bluing. These sections were then dehydrated in increasing concentrations of ethanol $(85 \%$, $95 \%$, and $100 \%$ for 5 min each) and transparentized with xylene. They were then mounted using neutral balsam, visualized under microscope, and photographed.

\section{Image analysis}

All digital images were acquired using a Motic AE31 fluorescence microscope (Motic China Group Co. Ltd, China) with a Moticam digital color camera (Motic China Group Co. Ltd). A total of three pictures were taken at random from each of the sections under the $\times 10$ and $\times 40$ objectives. The IHC stained tumor and RLH tissue section images were then evaluated using integrated optical density (IOD) and Image-Pro Plus software (version 6.0, Media Cybernetics Inc., USA). The IOD value was calculated by multiplying the optical density of the positive signals with the proportion of the positive area, which is indicated as the amount of corresponding protein in each image[28]. Each sample was evaluated using five independent measurements, and the final value for statistical analysis was presented as the mean IOD from all five measurements. A mean IOD value of more than 120 or 80 indicates high MCT1 or MCT4 expression, respectively. All observations and measurements were completed using coded slides, and the researchers performing these experiments were blind to the diagnosis in each of the tissue samples.

\section{Statistical analysis}

An unpaired t-test was used for comparing the mean IOD values of MCT1 and MCT4 and their differences in the various subgroups. The statistical significance of the correlations between high or low MCT1/MCT4 expression and survival time was estimated using the Kaplan-Meier method and a log-rank test. All statistical analyses were conducted and images were produced using IBM SPSS Statistics (version 21.0, SPSS Inc., USA) and GraphPad Prism (version 7.0, GraphPad Software Inc., USA). Statistical significance for two-tailed tests was set at $P \otimes 0.05$.

\section{Results}

\section{SLC16A1 and SLC16A3 expression analysis in TCL based on the CCLE data}

Analysis of the mRNA expression data from the RNAseq database in CCLE revealed that SLC16A1 (MCT1) expression was upregulated in T-cell lymphoma cell lines (Figure 1A), and SLC16A3 (MCT4) expression was also increased in all the cancer cell lines (Figure 1B).

\section{Patient demographics}

$28(73.7 \%)$ male and $10(26.3 \%)$ female patients with TCL were enrolled in this study, with the age at diagnosis being 18 to 87 years (median, 54 years). The number of patients categorized as Ann Arbor stages II II, III, and IV were 9, 6, 7, and 16, respectively. Of these, three presented bulky diseases. A total of 
22 patients exhibited extranodal involvement at diagnosis, and 15 demonstrated tumor progression over the course of their clinical follow-up. The duration of follow-up was 1-68 months (median, 28 months) and more detailed clinical information of each patient can be found in Supplemental Table 1.

\section{MCT1 and MCT4 expression in TCL}

The staining intensity for both the MCT1 and MCT4 proteins moving from low to high levels of expression in the neoplastic tissue membranes or cytoplasm is shown in Figure 2A-L. The IOD values for MCT1 and MCT4 were measured in both TCL (MCT1: range, 37.4 to 406.3, median, 123.0; MCT4: range, 22.4 to 292.3 , median, 84.2) and RLH tissue samples with the mean IOD values for both MCT1 and MCT4 being significantly higher in the TCL group when compared to the control (MCT1, $P=0.0392$, Figure 3A; MCT4, $P=0.0195$, Figure 3B).

Male patients had higher MCT1 expression than female patients $(P=0.0147)$, whereas the expression of MCT4 followed the opposite pattern $(P=0.0393$ ) (Table 1). Expression of MCT1 in TCL patients with an Ann Arbor stage of III-IV, increased serum LDH levels, and $>50 \% \mathrm{Ki}-67$ was significantly higher than in those with an Ann Arbor stage of $\mathbb{X}-I I(P=0.0375)$, normal serum LDH levels $(P=0.0308)$, and no more than $50 \% \mathrm{Ki}-67$ staining $(P=0.0116)$, respectively. However, there were no significant differences in MCT4 expression between any of these groups. There were no significant differences in MCT1 and MCT4 expression between patients when they were classified by age ( $>60$ years or not), performance status (ECOG score $>1$ or not), degree of extranodal involvement ( $\geq 2$ or not), bone marrow involvement, Serum $\beta 2-M G$ level (increased or normal), and the presence of "B" symptoms. 
Table 1

Comparisons of MCT1 and MCT4 expression in different TCL patient subgroups

\begin{tabular}{|c|c|c|c|c|c|}
\hline \multirow[t]{2}{*}{ Variable (number) } & & \multicolumn{2}{|l|}{ MCT1 } & \multicolumn{2}{|l|}{ MCT4 } \\
\hline & & Mean IOD & $P$ value & Mean IOD & $P$ value \\
\hline \multirow[t]{2}{*}{ Gender } & Male (28) & $114.7 \pm 13.3$ & \multirow[t]{2}{*}{0.0147} & $103.3 \pm 12.0$ & \multirow[t]{2}{*}{0.0393} \\
\hline & Female (10) & $179.4 \pm 19.7$ & & $59.5 \pm 5.5$ & \\
\hline \multirow[t]{2}{*}{ Age } & $\leq 60$ years $(22)$ & $144.0 \pm 18.8$ & \multirow[t]{2}{*}{0.2332} & $84.0 \pm 12.7$ & \multirow[t]{2}{*}{0.3403} \\
\hline & $>60$ years $(16)$ & $114.9 \pm 11.0$ & & $102.5 \pm 14.1$ & \\
\hline \multirow[t]{2}{*}{ Performance status (ECOG) } & $0-1(31)$ & $131.3 \pm 14.0$ & \multirow[t]{2}{*}{0.9444} & $91.7 \pm 11.2$ & \multirow[t]{2}{*}{0.9963} \\
\hline & $2-4(7)$ & $133.5 \pm 19.8$ & & $91.9 \pm 14.6$ & \\
\hline \multirow[t]{2}{*}{ Ann Arbor stage } & \-У (15) & $101.3 \pm 8.6$ & \multirow[t]{2}{*}{0.0375} & $105.0 \pm 14.9$ & \multirow[t]{2}{*}{0.2621} \\
\hline & $\mathbb{Q}-\mathbb{Q}(23)$ & $151.5 \pm 17.9$ & & $83.1 \pm 12.1$ & \\
\hline \multirow[t]{2}{*}{ Serum LDH } & Normal (18) & $104.9 \pm 11.5$ & \multirow[t]{2}{*}{0.0308} & $98.5 \pm 12.9$ & \multirow[t]{2}{*}{0.5030} \\
\hline & Increased (20) & $155.9 \pm 18.8$ & & $85.7 \pm 13.8$ & \\
\hline \multirow[t]{2}{*}{ Extranodal involvement } & $<2(25)$ & $123.8 \pm 10.5$ & \multirow[t]{2}{*}{0.3648} & $91.0 \pm 11.0$ & \multirow[t]{2}{*}{0.9153} \\
\hline & $\geq 2(13)$ & $146.9 \pm 28.8$ & & $93.2 \pm 18.5$ & \\
\hline \multirow[t]{2}{*}{ Bone marrow involvement } & Yes (14) & $125.5 \pm 15.7$ & \multirow[t]{2}{*}{0.6949} & $89.3 \pm 10.7$ & \multirow[t]{2}{*}{0.8438} \\
\hline & No (24) & $135.4 \pm 16.7$ & & $93.2 \pm 13.8$ & \\
\hline \multirow[t]{2}{*}{ Ki-67 index } & $\leq 50 \%(19)$ & $102.3 \pm 10.8$ & \multirow[t]{2}{*}{0.0116} & $96.8 \pm 12.3$ & \multirow[t]{2}{*}{0.6001} \\
\hline & $>50 \%$ (19) & $161.1 \pm 19.3$ & & $86.7 \pm 14.6$ & \\
\hline \multirow[t]{2}{*}{ Serum $\beta 2-M G$} & Normal (11) & $106.0 \pm 11.5$ & \multirow[t]{2}{*}{0.1717} & $92.6 \pm 19.6$ & \multirow[t]{2}{*}{0.9544} \\
\hline & Increased (27) & $142.2 \pm 15.8$ & & $91.4 \pm 10.9$ & \\
\hline \multirow[t]{2}{*}{ “B” symptoms } & Present (16) & $128.5 \pm 17.3$ & \multirow[t]{2}{*}{0.8197} & $86.9 \pm 11.1$ & \multirow[t]{2}{*}{0.6649} \\
\hline & Absent (22) & $134.1 \pm 16.7$ & & $95.3 \pm 14.3$ & \\
\hline
\end{tabular}

The mean IOD values for MCT1 and MCT4 in patients diagnosed with the three dominate pathological types (PTCL, NOS, AITL, and ENKTL) are summarized in Figure $3 C$ and D, and no significant differences in MCT expression were noted for any of them. Each patient was also assigned an IPI and was classified into low-risk (0-1), intermediate-risk (2-3), or high-risk (4-5) group based on the score. MCT1 expression was significantly lower in the low-risk group when compared with either the intermediate-risk $(P=0.0323)$ 
or high-risk ( $\mathrm{P}=0.0401)$ groups (Figure $3 \mathrm{E})$, while MCT4 expression was not significantly different between these groups (Figure $3 \mathrm{~F}$ ).

\section{Prognostic value of MCT1 and MCT4}

Univariate survival analysis of patients with TCL in relation to MCT1 and MCT4 expression is shown in Figure 4. Patients with high levels of MCT1 exhibited reduced OS $(P=0.0475)$ and $P F S(P=0.0246)$ rates, as determined by the log-rank test. However, we did not find any significant differences in the OS or PFS in patients with varying levels of MCT4 expression.

\section{Discussion}

The use of IHC, cytogenetics, and molecular evaluations have had a significant impact on the diagnostic accuracy and subtype assignment of $\operatorname{TCL}[29,30]$. However, there is still no universally accepted standard treatment for TCL patients despite the concentrated efforts to facilitate this over the last few decades, resulting in our deeper understanding of these diseases[31, 32]. New targeted therapies for TCL are constantly emerging because patients with TCL exhibit higher rate of failure and relapse in response to first-line treatments as compared to that seen in B cell lymphomas. A variety of target-specific treatments are undergoing clinical trials and are expected to help improve prognosis in patients with TCL. These interventions were designed based on the following mechanisms[33, 34]: (1) Epigenetic regulation, mainly consisting of histone deacetylase inhibitors (HDACi), like Vorinostat, Belinostat, Romidepsin, Panobinostat, Chidamide, Quisinostat, and AR-42[35]; (2) Antibody dependent cell-mediated cytotoxicity (ADCC), including technologies like Brentuximab vedotin (targeted CD30), Daratumumab (targeted CD38), Alemtuzumab (targeted CD52), IPH4102[36] (targeted KIR3DL2), TTI-621[37] (targeted CD47), and AFM13[38] (targeted CD30/CD16A); (3) Cytotoxic reactions, involving chimeric antigen receptor T or NK (CAR-T/CAR-NK) cells with anti-CD7, anti-CD4, anti-CD5 or anti-TCR capabilities; (4) Signaling pathway blockers, including anaplastic lymphoma kinase (ALK) inhibitors, phosphatidylinositol 3-kinase (PI3K) inhibitors, and microRNA (miR)-155 inhibitors[39]; (5) Other agents, such as E7777[40] (an immunotoxin targeting the interleukin (IL)-2 receptor), Alisertib[41] (an inhibitor of Aurora A kinase (AAK)), and various antibiotics from Staphylococcus aureus[42] amongst others. However, to date, none of these technologies have produced a satisfactory outcome in patients with $\mathrm{TCL}$, suggesting that further interventions are needed.

Recently, insights into tumor lactic acid metabolism, especially the transporters on the cell membrane, have generated meaningful breakthroughs about MCTs, resulting in several ongoing studies evaluating the inhibition of the MCTs or the mechanisms underlying MCT function and their roles in oncogenesis and progression. Lactate is the major substrate for energy production in cancer cells and acts as a signaling molecule in these cells. It has also been shown to play important roles in tumor progression, including inducing tumor angiogenesis, inhibiting histone deacetylases, stimulating amino acid metabolism, activating lactate receptor GPR81, and inducing tumor immune tolerance[43]. Therefore, MCTs, which control the transmembrane flux of lactate, have become targets of rigorous evaluation in various types of cancers. Doherty[44] et al. showed that disrupting the function of MCT1 can lead to an 
accumulation of intracellular lactate and tumor cell death, while Baek et al. reported that MCT4 depletion induces cell death, which is characterized by elevated reactive oxygen species and metabolic crisis[45]. Pértega-Gomes et al. believed that MCT2 should be explored as a tumor marker[46] and several studies have evaluated the expression levels of various MCTs in different cancers and shown that they share a similar pattern of dysregulation in various cancers. However, their expression in TCLs is largely undefined. Our study evaluated MCT expression in TCL, and revealed that MCT1 and MCT4 exhibit high expression levels in tissue samples from patients with TCL. These results are consistent with the open-access data for SLC16A1 and SLC16A3 reported in the CCLE. To the best of our knowledge, MCT1 has also been found to be highly expressed in lung cancer[47], breast cancer[47, 48], brain cancer[49], cervix cancer[15], skin cancer[16], soft tissue cancer[50], and B cell lymphomas[24]. However, Noble et al. reported that little to no MCT4 protein was present in most patients with diffuse large B-cell lymphoma (DLBCL) and Burkitt lymphoma (BL)[24], and this contradicts with our results in patients with TCL. AZD3965, a selective inhibitor of MCT1, is under phase I clinical trials for use in adult solid tumors and two types of B-cell lymphoma, DLBCL and BL[19]. Likewise, as a subgroup of the non-Hodgkin lymphomas, TCLs are likely to respond to MCT1 inhibition and thus benefit from these new inhibitors, even though the incidence of TCL is much lower. That said there is still a long way to go in linking MCT1 targeted treatment with improved outcomes in patients with TCL, including their evaluation in animal models. But before that, we need to establish whether increased MCT1 expression affects the prognosis of patients with TCL.

Our study may provide a preliminary answer to this question. Survival analyses indicated that TCL patients with high levels of MCT1 expression were more likely to have shorter OS and PFS, while those with high MCT4 expression did not exhibit any significant differences in these parameters. Similarly, our evaluation of the prognostic significance of MCT1 and MCT4 demonstrated similar results to those of a previous study in head and neck cancer samples[51]. However, this was not the case with several other types of cancers, as high levels of MCT4 have been linked to poor prognosis in patients with prostate cancer[46], pancreatic cancer[45], lung adenocarcinoma[52], and non-small cell lung cancer (NSCLC)[53]. Moreover, we found that high levels of MCT1 was more common in female patients, advanced stages of disease, in tissues with increased serum LDH and a Ki-67 index of more than $50 \%$ than their counterparts. These characteristics, except for sex, are also already considered risk factors for patients with $\mathrm{NHL}[54$, 55]. Interestingly, we found that female patients with TCL presented with higher MCT1 but lower MCT4 expression than their male counterparts, but this needs to be verified using a larger sample size in the future. We also completed a series of comparisons using a risk-based stratification method which revealed that patients in the intermediate-and high-risk groups also exhibited higher MCT1 protein expression levels than those in the low-risk group. Thus we speculate that high MCT1 expression is related to poor prognosis in patients with TCL, although the mechanism of this remains unknown.

Notably, the differences in MCT1 expression between subgroups were generally more apparent than the differences in MCT4 expression. Although this does not mean MCT1 is more valuable than MCT4 in TCL prognosis, we can conclude that high expression levels of MCT1 can be considered a considerable risk factor in these patients. We did not observe any significant differences in MCT1 or MCT4 expression between TCL subtypes (confined to PTCL-NOS, AITL, and ENKTL), and further studies should evaluate 
this in more detail for some of the rarer subtypes. Currently, out of all the lymphomas, only DLBCL and BL are currently implicated in any of the clinical trials for selective inhibitor AZD3965, which inhibits MCT1. Previous studies have not compared MCT1 expression levels in these two NHL subtypes nor explicitly proposed which is more amenable to AZD3965[24, 25]. In the case of DLBCL, two related studies have both revealed that MCT1 expression is increased in patient tumor samples and that this increased expression is associated with poor prognosis. However, evaluation of MCT4 expression revealed a distinct difference in the positivity rates for these two studies, reporting $27 \%$ and $65.6 \%$ positivity respectively. Afonso et al. concluded that MCT1 might serve as a target for treating patients with NHL (or DLBCL) with high MCT1/low MCT4 expression[25]. This means that our data, which showed that TCL samples can present with increased expression of both MCT1 and MCT4, does not meet this criterion. However, this literature also suggests that these results were obtained in vitro and need to be confirmed in additional studies designed to evaluate novel therapeutic strategies. Our data suggests that TCL may be amenable to treatment with MCT1 selective inhibitors, but this requires preclinical validation both in vitro and in vivo.

In summary, we analyzed the expression of MCT1 and MCT4 in TCL using IHC and explored their prognostic value in relation to specific clinical parameters. We found that both MCT1 and MCT4 were highly expressed in the pathological tissues of TCL patients, and that high MCT1 expression may be associated with poor OS and PFS in these patients. Our results also indicate that patients with TCL in the high-and intermediate-risk groups exhibit higher MCT1 expression levels than those in the low-risk group. In addition, high MCT1 expression is also more common in female patients and those with advanced stage disease, increased serum LDH levels and $\mathrm{Ki}-67$ at $\geq 50 \%$. Taken together this data suggests that MCT1 inhibition may be a valid choice for treating TCL, but more data from larger populations and preclinical studies are required to confirm this hypothesis.

\section{Declarations}

\section{Funding}

This study was supported by Hunan Provincial Science and Technology Department, China.

\section{Competing interests}

There are no competing interests to declare.

\section{Availability of data and material}

The data from this study are available from the corresponding author upon reasonable request.

\section{Authors' Contributions}

$\mathrm{HZ}$ drafted the manuscript, $\mathrm{HZ}, \mathrm{GH}$, and $\mathrm{YL}$ were responsible for conceiving the study, $\mathrm{HZ}, \mathrm{YC}, \mathrm{QY}, \mathrm{JL}, \mathrm{YX}$, and $\mathrm{HC}$ analyzed and interpreted the data, $\mathrm{HZ}$ performed the immunohistochemical staining and 
evaluations, ZC, LY, and HC managed and followed up the patients, GH, YL, and YC made various critical revisions to the manuscript, $\mathrm{GH}, \mathrm{YC}$, and $\mathrm{YL}$ contributed to study design, $\mathrm{HZ}$ conceived the original idea and $\mathrm{GH}$ supervised the project. All authors approved the final version of the manuscript prior to submission.

\section{Acknowledgements}

The authors thank their colleagues in the Hematology Laboratory at The Affiliated Zhuzhou Hospital, XiangYa School of Medicine, Central South University, for their advice and assistance during these studies.

\section{References}

1. Vose J, Armitage J, Weisenburger D. International peripheral T-cell and natural killer/T-cell lymphoma study: pathology findings and clinical outcomes. Journal of clinical oncology : official journal of the American Society of Clinical Oncology. 2008,26(25):4124-30.

2. Perry A, Diebold J, Nathwani B, et al. Non-Hodgkin lymphoma in the developing world: review of 4539 cases from the International Non-Hodgkin Lymphoma Classification Project. Haematologica. 2016,101(10):1244-50.

3. Perry A, Diebold J, Nathwani B, et al. Non-Hodgkin lymphoma in the Far East: review of 730 cases from the international non-Hodgkin lymphoma classification project. Annals of hematology. 2016,95(2):245-51.

4. Sun J, Yang Q, Lu Z, et al. Distribution of lymphoid neoplasms in China: analysis of 4,638 cases according to the World Health Organization classification. American journal of clinical pathology. 2012,138(3):429-34.

5. Swerdlow S, Campo E, Pileri S, et al. The 2016 revision of the World Health Organization classification of lymphoid neoplasms. Blood. 2016,127(20):2375-90.

6. Briski R, Feldman A, Bailey N, et al. The role of front-line anthracycline-containing chemotherapy regimens in peripheral T-cell lymphomas. Blood cancer journal. 2014,4:e214.

7. Abouyabis A, Shenoy P, Sinha R, Flowers C, Lechowicz M. A Systematic Review and Meta-Analysis of Front-line Anthracycline-Based Chemotherapy Regimens for Peripheral T-Cell Lymphoma. ISRN hematology. 2011,2011:623924.

8. Mak V, Hamm J, Chhanabhai M, et al. Survival of patients with peripheral T-cell lymphoma after first relapse or progression: spectrum of disease and rare long-term survivors. Journal of clinical oncology : official journal of the American Society of Clinical Oncology. 2013,31(16):1970-6.

9. Kim S, Yoon S, Suzuki R, et al. Comparison of outcomes between autologous and allogeneic hematopoietic stem cell transplantation for peripheral T-cell lymphomas with central review of pathology. Leukemia. 2013,27(6):1394-7.

10. Warburg O. On the origin of cancer cells. Science (New York, N.Y.). 1956,123(3191):309-14. 
11. Lunt S, Vander Heiden M. Aerobic glycolysis: meeting the metabolic requirements of cell proliferation. Annual review of cell and developmental biology. 2011,27:441-64.

12. Halestrap A. The monocarboxylate transporter family-Structure and functional characterization. IUBMB life. 2012,64(1):1-9.

13. Halestrap A, Wilson M. The monocarboxylate transporter family--role and regulation. IUBMB life. 2012,64(2):109-19.

14. Pérez-Escuredo J, Van Hée V, Sboarina M, et al. Monocarboxylate transporters in the brain and in cancer. Biochimica et biophysica acta. 2016,1863(10):2481-97.

15. Pinheiro $C$, Longatto-Filho A, Ferreira L, et al. Increasing expression of monocarboxylate transporters 1 and 4 along progression to invasive cervical carcinoma. International journal of gynecological pathology : official journal of the International Society of Gynecological Pathologists. 2008,27(4):568-74.

16. Ho J, de Moura M, Lin Y, et al. Importance of glycolysis and oxidative phosphorylation in advanced melanoma. Molecular cancer. 2012,11:76.

17. Sonveaux P, Végran F, Schroeder T, et al. Targeting lactate-fueled respiration selectively kills hypoxic tumor cells in mice. The Journal of clinical investigation. 2008,118(12):3930-42.

18. Semenza G. Tumor metabolism: cancer cells give and take lactate. The Journal of clinical investigation. 2008,118(12):3835-7.

19. Bola B, Chadwick A, Michopoulos F, et al. Inhibition of monocarboxylate transporter-1 (MCT1) by AZD3965 enhances radiosensitivity by reducing lactate transport. Molecular cancer therapeutics. 2014,13(12):2805-16.

20. Beloueche-Babari M, Wantuch S, Casals Galobart T, et al. MCT1 Inhibitor AZD3965 Increases Mitochondrial Metabolism, Facilitating Combination Therapy and Noninvasive Magnetic Resonance Spectroscopy. Cancer research. 2017,77(21):5913-24.

21. Curtis N, Mooney L, Hopcroft L, et al. Pre-clinical pharmacology of AZD3965, a selective inhibitor of MCT1: DLBCL, NHL and Burkitt's lymphoma anti-tumor activity. Oncotarget. 2017,8(41):69219-36.

22. Braga M, Kaliszczak M, Carroll L, et al. Tracing Nutrient Flux Following Monocarboxylate Transporter1 Inhibition with AZD3965. Cancers. 2020,12(6).

23. Beloueche-Babari M, Casals Galobart T, Delgado-Goni T, et al. Monocarboxylate transporter 1 blockade with AZD3965 inhibits lipid biosynthesis and increases tumour immune cell infiltration. British journal of cancer. 2020,122(6):895-903.

24. Noble R, Bell N, Blair $\mathrm{H}$, et al. Inhibition of monocarboxyate transporter 1 by AZD3965 as a novel therapeutic approach for diffuse large B-cell lymphoma and Burkitt lymphoma. Haematologica. 2017,102(7):1247-57.

25. Afonso J, Pinto T, Simões-Sousa S, et al. Clinical significance of metabolism-related biomarkers in non-Hodgkin lymphoma - MCT1 as potential target in diffuse large B cell lymphoma. Cellular oncology (Dordrecht). 2019,42(3):303-18. 
26. Ghandi M, Huang F, Jané-Valbuena J, et al. Next-generation characterization of the Cancer Cell Line Encyclopedia. Nature. 2019,569(7757):503-08.

27. Cheson B, Fisher R, Barrington S, et al. Recommendations for initial evaluation, staging, and response assessment of Hodgkin and non-Hodgkin lymphoma: the Lugano classification. Journal of clinical oncology : official journal of the American Society of Clinical Oncology. 2014,32(27):3059-68.

28. Gao S, Zhang T, Jin L, et al. CAPON Is a Critical Protein in Synaptic Molecular Networks in the Prefrontal Cortex of Mood Disorder Patients and Contributes to Depression-Like Behavior in a Mouse Model. Cerebral cortex (New York, N.Y. : 1991). 2019,29(9):3752-65.

29. Foss FM, Zinzani PL, Vose JM, Gascoyne RD, Rosen ST, Tobinai K. Peripheral T-cell lymphoma. Blood. 2011,117(25):6756-67.

30. Pizzi M, Margolskee E, Inghirami G. Pathogenesis of Peripheral T Cell Lymphoma. Annu Rev Pathol. 2018,13:293-320.

31. Casulo C, O'Connor O, Shustov A, et al. T-Cell Lymphoma: Recent Advances in Characterization and New Opportunities for Treatment. J Natl Cancer Inst. 2017,109(2).

32. Phan A, Veldman R, Lechowicz MJ. T-cell Lymphoma Epidemiology: the Known and Unknown. Curr Hematol Malig Rep. 2016,11(6):492-503.

33. Izykowska K, Rassek K, Korsak D, Przybylski GK. Novel targeted therapies of T cell lymphomas. J Hematol Oncol. 2020,13(1):176.

34. Ito Y, Makita S, Tobinai K. Development of new agents for peripheral T-cell lymphoma. Expert Opin Biol Ther. 2019,19(3):197-209.

35. Sborov D, Canella A, Hade E, et al. A phase 1 trial of the HDAC inhibitor AR-42 in patients with multiple myeloma and T- and B-cell lymphomas. Leukemia \& lymphoma. 2017,58(10):2310-18.

36. Bagot M, Porcu P, Marie-Cardine A, et al. IPH4102, a first-in-class anti-KIR3DL2 monoclonal antibody, in patients with relapsed or refractory cutaneous T-cell lymphoma: an international, first-in-human, open-label, phase 1 trial. The Lancet. Oncology. 2019,20(8):1160-70.

37. Ansell S, Maris M, Lesokhin A, et al. Phase I Study of the CD47 Blocker TTI-621 in Patients with Relapsed or Refractory Hematologic Malignancies. Clinical cancer research : an official journal of the American Association for Cancer Research. 2021,27(8):2190-99.

38. Rothe A, Sasse S, Topp M, et al. A phase 1 study of the bispecific anti-CD30/CD16A antibody construct AFM13 in patients with relapsed or refractory Hodgkin lymphoma. Blood. 2015,125(26):4024-31.

39. Seto A, Beatty X, Lynch J, et al. Cobomarsen, an oligonucleotide inhibitor of miR-155, co-ordinately regulates multiple survival pathways to reduce cellular proliferation and survival in cutaneous T-cell lymphoma. British journal of haematology. 2018,183(3):428-44.

40. Kawai H, Ando K, Maruyama D, et al. Phase II study of E7777 in Japanese patients with relapsed/refractory peripheral and cutaneous T-cell lymphoma. Cancer science. 2021,112(6):242635. 
41. Pusalkar S, Zhou X, Li Y, et al. Biotransformation Pathways and Metabolite Profiles of Oral [C]Alisertib (MLN8237), an Investigational Aurora A Kinase Inhibitor, in Patients with Advanced Solid Tumors. Drug metabolism and disposition: the biological fate of chemicals. 2020,48(3):217-29.

42. Lindahl L, Willerslev-Olsen A, Gjerdrum L, et al. Antibiotics inhibit tumor and disease activity in cutaneous T-cell lymphoma. Blood. 2019,134(13):1072-83.

43. Payen V, Mina E, Van Hée V, Porporato P, Sonveaux P. Monocarboxylate transporters in cancer. Molecular metabolism. 2020,33:48-66.

44. Doherty J, Yang C, Scott K, et al. Blocking lactate export by inhibiting the Myc target MCT1 Disables glycolysis and glutathione synthesis. Cancer research. 2014,74(3):908-20.

45. Baek G, Tse Y, Hu Z, et al. MCT4 defines a glycolytic subtype of pancreatic cancer with poor prognosis and unique metabolic dependencies. Cell reports. 2014,9(6):2233-49.

46. Pértega-Gomes N, Vizcaíno J, Miranda-Gonçalves V, et al. Monocarboxylate transporter 4 (MCT4) and CD147 overexpression is associated with poor prognosis in prostate cancer. BMC cancer. 2011,11:312.

47. Pinheiro C, Reis R, Ricardo S, Longatto-Filho A, Schmitt F, Baltazar F. Expression of monocarboxylate transporters 1, 2, and 4 in human tumours and their association with CD147 and CD44. Journal of biomedicine \& biotechnology. 2010,2010:427694.

48. Pinheiro C, Albergaria A, Paredes J, et al. Monocarboxylate transporter 1 is up-regulated in basal-like breast carcinoma. Histopathology. 2010,56(7):860-7.

49. Miranda-Gonçalves V, Honavar M, Pinheiro C, et al. Monocarboxylate transporters (MCTs) in gliomas: expression and exploitation as therapeutic targets. Neuro-oncology. 2013,15(2):172-88.

50. Zhao Z, Wu M, Zou C, et al. Downregulation of MCT1 inhibits tumor growth, metastasis and enhances chemotherapeutic efficacy in osteosarcoma through regulation of the NF-KB pathway. Cancer letters. 2014,342(1):150-8.

51. Leu M, Kitz J, Pilavakis Y, et al. Monocarboxylate transporter-1 (MCT1) protein expression in head and neck cancer affects clinical outcome. Scientific reports. 2021,11(1):4578.

52. Ruan Y, Zeng F, Cheng Z, Zhao X, Fu P, Chen H. High expression of monocarboxylate transporter 4 predicts poor prognosis in patients with lung adenocarcinoma. Oncology letters. 2017,14(5):5727-34.

53. Kuo T, Huang K, Yang S, et al. Monocarboxylate Transporter 4 Is a Therapeutic Target in Non-small Cell Lung Cancer with Aerobic Glycolysis Preference. Molecular therapy oncolytics. 2020,18:189-201.

54. International_Non-Hodgkin's_Lymphoma_Prognostic_Factors_Project. A predictive model for aggressive non-Hodgkin's lymphoma. N Engl J Med. 1993,329(14):987-94.

55. Szczuraszek K, Mazur G, Jeleń M, Dziegiel P, Surowiak P, Zabel M. Prognostic significance of Ki-67 antigen expression in non-Hodgkin's lymphomas. Anticancer research. 2008,28:1113-8.

\section{Figures}



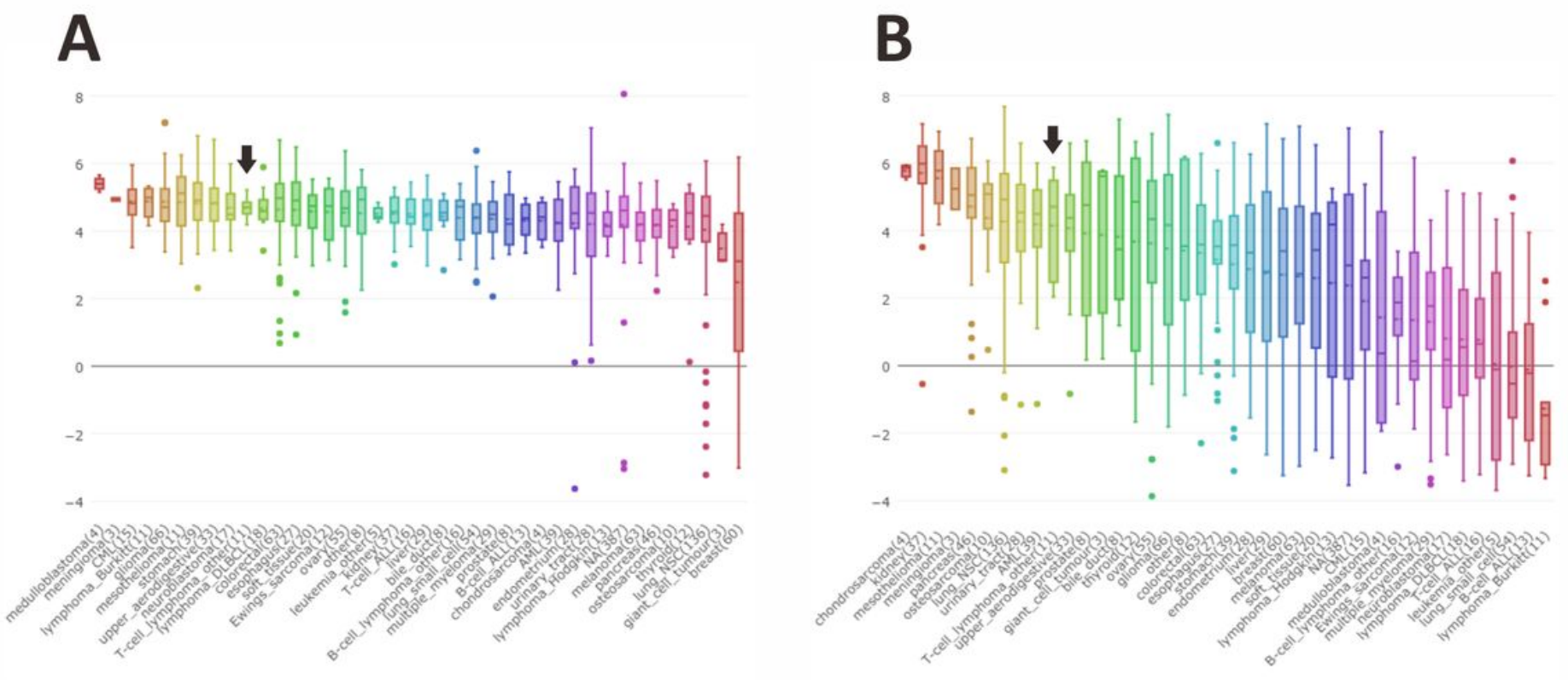

Figure 1

Expression of the MCT1 and MCT4 mRNA transcripts in cancer cell lines. The black arrows indicate the boxes representing the normalized expression levels of these genes in T-cell lymphoma cell lines. (A) MCT1 (SLC16A1) expression in cancer cell lines. (B) MCT4 (SLC16A3) expression in cancer cell lines. All data was obtained from the Cancer Cell Line Encyclopaedia (CCLE). 
MCT1

Mean IOD=37.4

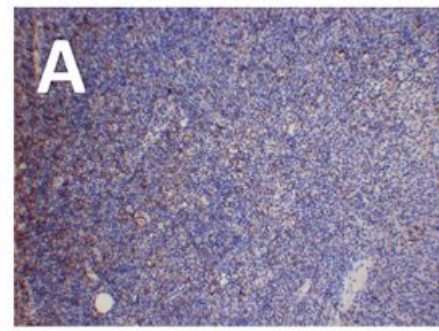

$10 x$

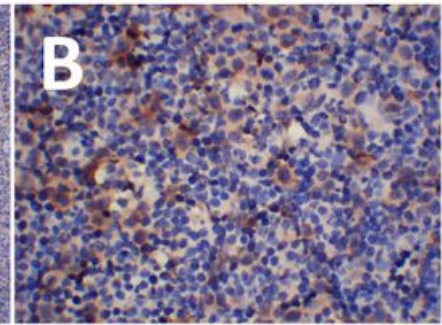

$40 x$

Mean IOD=122.9

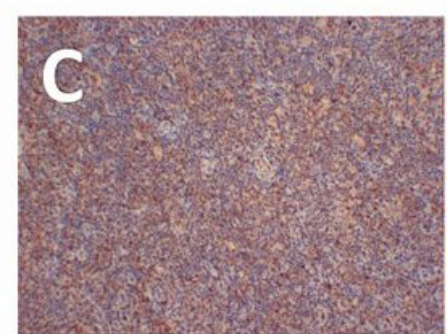

$10 x$

Mean IOD $=242.3$

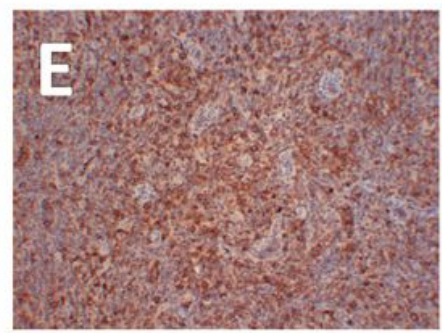

$10 x$

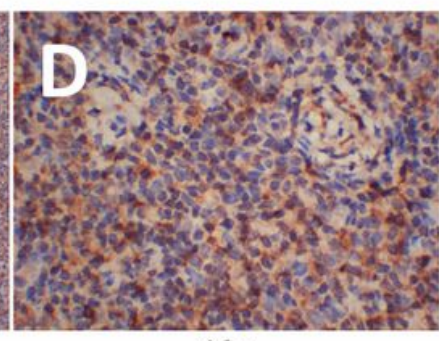

$40 x$

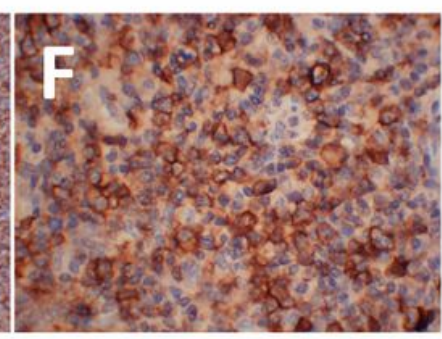

$40 x$

MCT4

Mean IOD=22.4

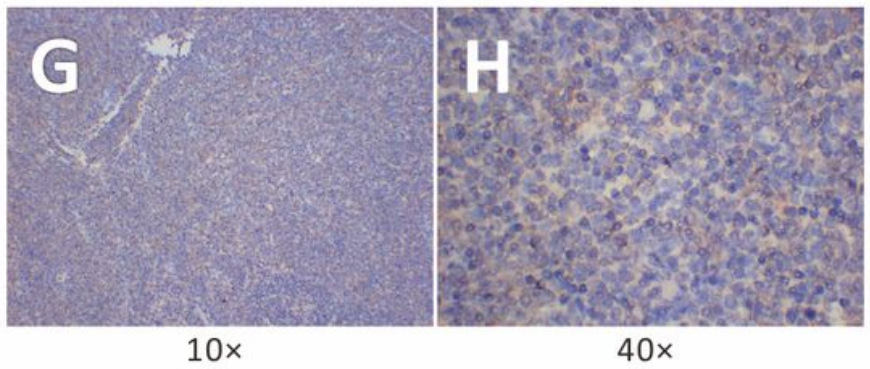

Mean IOD=81.8

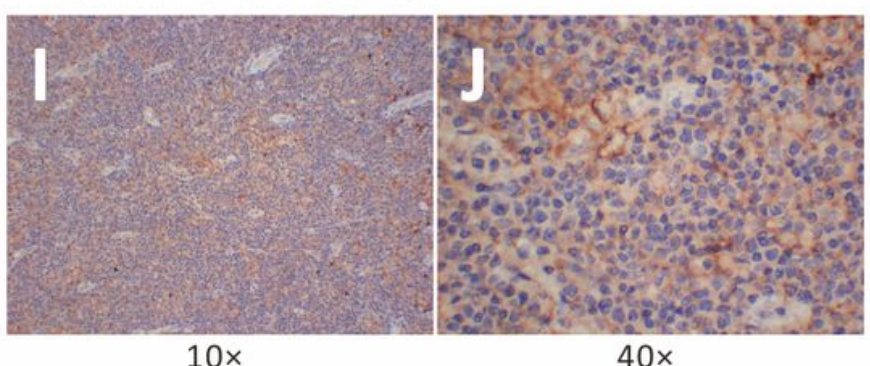

Mean IOD=149.6

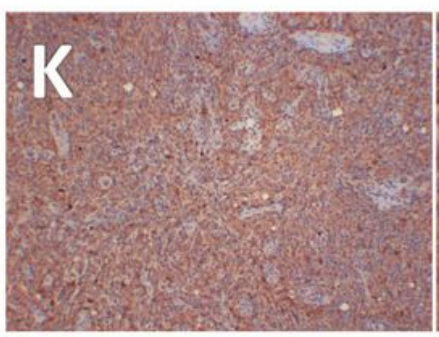

$10 x$

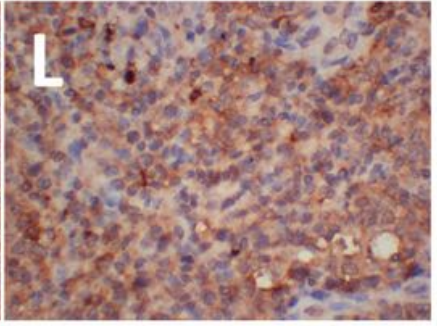

$40 x$

Figure 2

Immunohistochemical staining for MCT1 and MCT4 in TCL tissue samples. Staining intensity is presented as the mean integrated optical density (IOD). The panels display representative images of MCT1 and MCT4 expression in each of the TCL tissue samples moving from low levels of expression through moderate expression and ending in high expression samples. These sections are shown under both $\times 10$ and $\times 40$ magnification. 
A

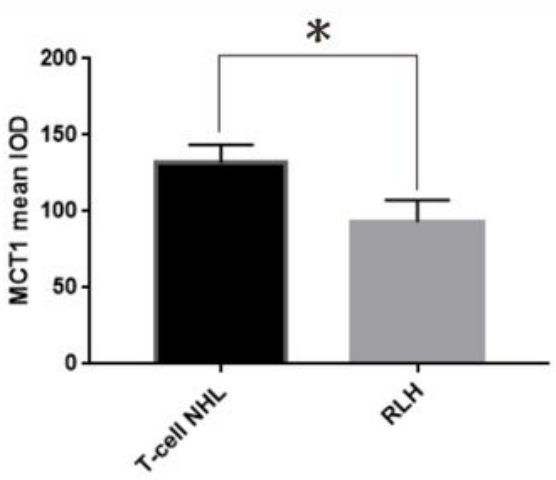

C

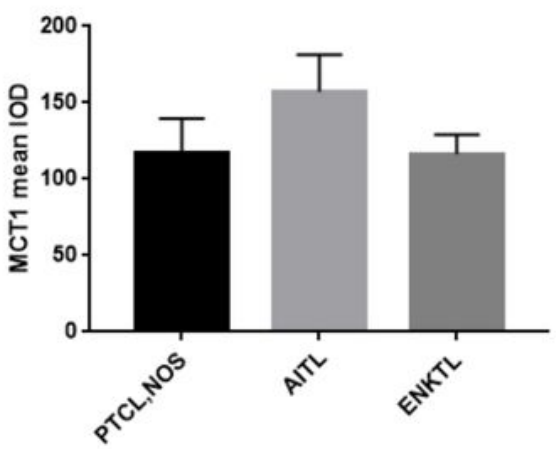

E

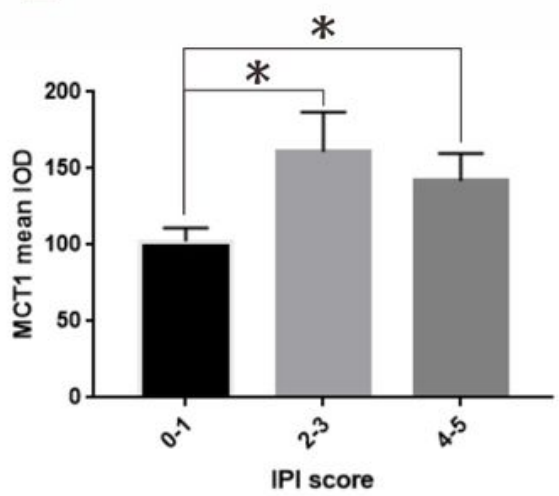

B

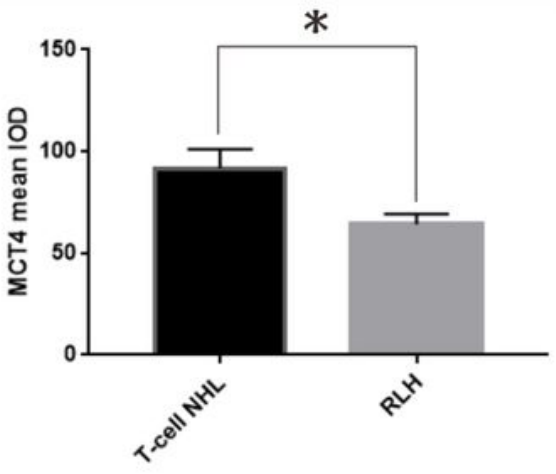

D

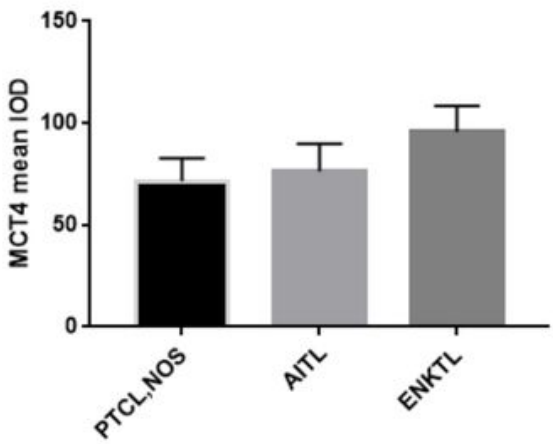

$\mathbf{F}$

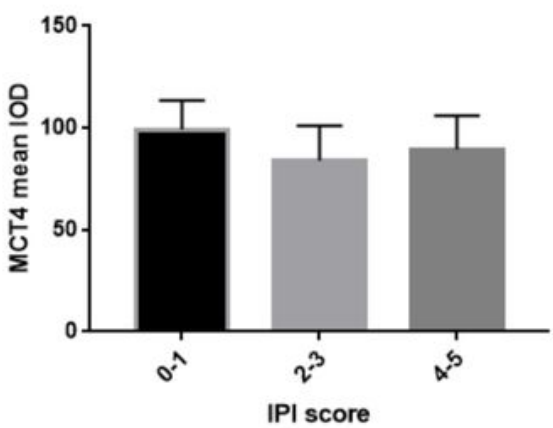

Figure 3

Comparisons of MCT1 and MCT4 expression in TCL tissues. (A) MCT1 expression in patients with T-cell non-Hodgkin lymphoma $(\mathrm{NHL})$ and reactive lymphoid hyperplasia $(\mathrm{RLH}$, which serve as the control group). (B) MCT4 expression in patients with T-cell NHL and RLH. (C) MCT1 expression in patients diagnosed with peripheral T-cell lymphoma, not otherwise specified (PTCL, NOS), angioimmunoblastic Tcell lymphoma (AITL), and extranodal NK/T-cell lymphoma, nasal type (ENKTL). (D) MCT4 expression in 
patients diagnosed with PTCL, NOS, AITL, and ENKTL. (E) MCT1 expression in T-cell NHL patients with International Prognostic Index (IPI) scores of 0-1, 2-3, and 4-5. (F) MCT4 expression in T-cell NHL patients with IPI scores of 0-1, 2-3, and 4-5. Graphs show the means from five independent measurements \pm SEM. * $\mathrm{P}<0.05$ as determined by an unpaired two-tailed t-test.
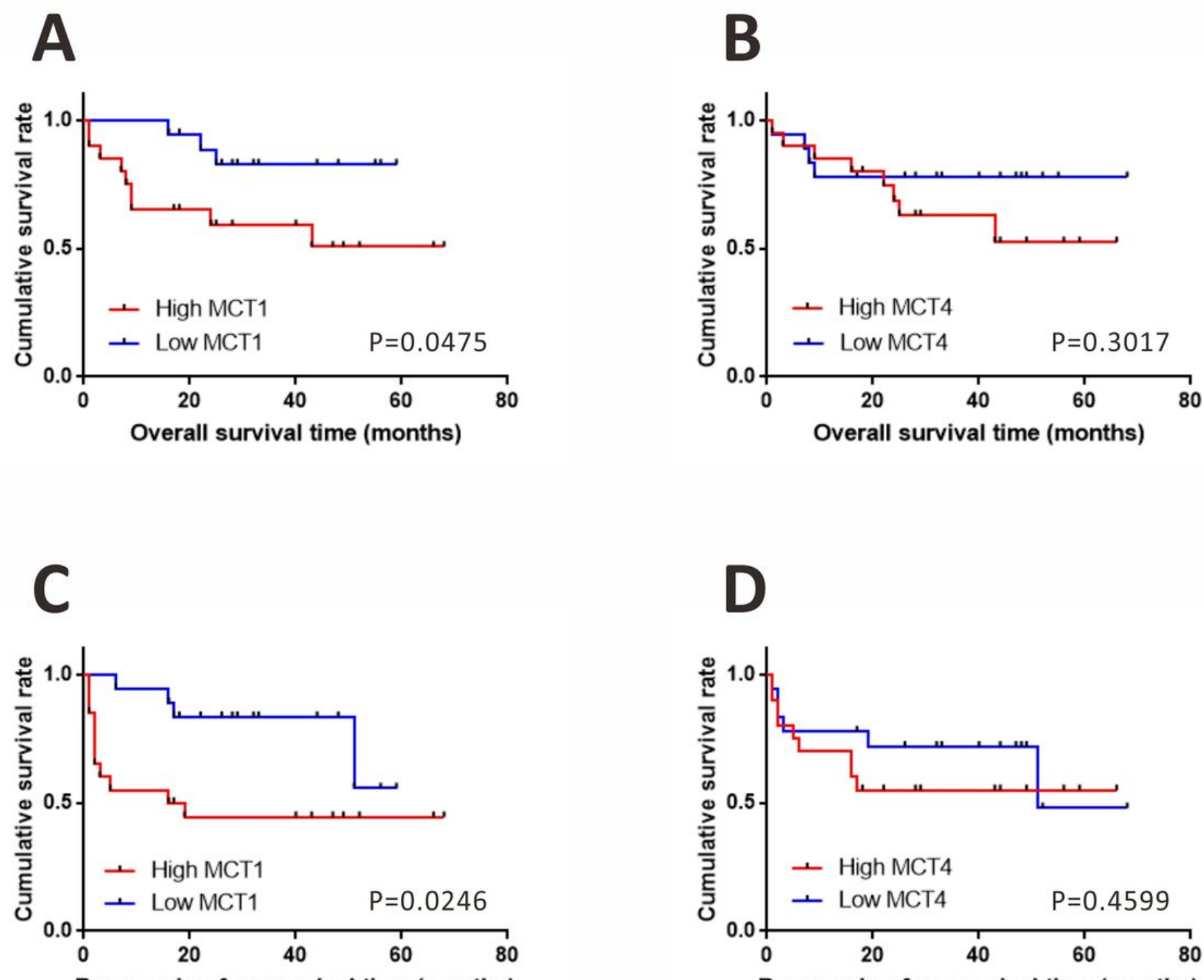

Progression-free survival time (months)

Progression-free survival time (months)

Figure 4

Kaplan-Meier survival analysis of T-cell lymphoma (TCL) patients stratified according to their MCT1 and MCT 4 expression levels. (A) Survival curves for the overall survival (OS) of TCL patients with low and high levels of MCT1. (B) Survival curves for the OS of TCL patients with low and high levels of MCT4. (C) Survival curves for progression-free survival (PFS) in TCL patients with low and high levels of MCT1. (D) Survival curves for PFS in TCL patients with low and high levels of MCT4.

\section{Supplementary Files}


This is a list of supplementary files associated with this preprint. Click to download.

- SupplementaryTable1.xlsx 